\title{
Characterization of plasma formation and mass ejection in Exploding Foil Initiators
}

\author{
Alexander J. Borman and Colin F. Dowding
}

\begin{abstract}
To aid exploding foil initiator (EFI) design, better prediction of ejecta momentum through either mass or velocity prediction is required. A numerical model was developed to calculate the mass of material converted to plasma within the confined region of an exploding foil initiator bridge during change of state under an electrical stimulus from a discharging capacitor. Optimisation is facilitated through the increased understanding of plasma evolution in current EFI designs, including the impact of this on both current delivery to the bridge and overall unit efficiency. The plasma regions were formed in key regions within the bridge, termed $P_{A}$ (ground side of EFI) and $P_{B}$ (high-voltage side of EFI) in this work. Different regions were dominant in mass ejection for different operating voltages. A trend is identified wherein the bridge exhibits an optimum threshold between the capacitor energy being utilized for mass conversion to plasma and that used for acceleration of this mass. It is postulated that, through geometric design modification, this threshold can be adjusted to deliver the momentum threshold of the explosive for which an EFI may be designed.
\end{abstract}

Index Terms-Exploding Foil Initiator; Finite Element Modelling; Plasma Mass Ejecta

\section{INTRODUCTION}

$\mathrm{E}$ xploding foil initiators (EFI) were developed in the 1970's, at Lawrence Livermore National Laboratory [1] as a means of explosive initiation whilst being comparatively less sensitive to external influences such as electrostatics than other means of initiation.

The EFI has a laminar structure [2] consisting of a tamper, bridge, flyer layer and barrel; some units are manufactured with a supply strip-line incorporated, some are manufactured separately [3].

It is a means of explosive initiation operated by conversion of an electrical stimuli from capacitor discharge to a mechanical impulse of ejecta from the unit. Electrical energy stored in the high-voltage capacitor within the capacitor discharge circuit is discharged through a thin, shaped bridge causing a rapid state change from solid through to ionized plasma. Increasing temperatures and pressures, aided by the bridge geometry, from these rapid changes of state, form a plasma that drives a proportion of the insulated flyer layer upwards [4].

Submitted for review on $13^{\text {th }}$ February 2020 , revised $28^{\text {th }}$ July 2020 . The Authors wish to thank Teledyne-e2 $\mathrm{v}$ for their support in the completion of project from which this paper has come.

A. J. Borman is with the University of Lincoln, Brayford Pool, Lincoln, LN6 7TS, UK (e-mail: aborman@ lincoln.ac.uk).
The specific geometry of the bridge is variable, this geometry influences EFI operation. During operation, discussed below, a series of changes of state take place within this layer converting it from a solid to a plasma.

The most common materials used for bridge construction are either aluminium or copper, chosen for their suitable electrical burst qualities [1]. Readily available copper-Kapton laminates (from DuPont [5]) make copper a material of choice for smaller scale EFI units, Kapton being a primary choice for flyer layer material [6].

Considering the electrical stimuli delivered through the EFI, [7] observed that, at high enough initial capacitor voltages, the current discharged through the EFI bridge was saturated at a lower level than for reduced initial capacitor voltages for the same bridge geometry.

The EFI utilizes shock initiation to trigger explosion of an explosive material located on top of the unit and impacted on by the ejecta [8]. The explosive pellet's behaviour is directly related to shock pressure [8], whether the pressure wave developed within the explosive material can reach and sustain sufficient velocity to move from deflagration to detonation [9]. A threshold velocity for this transition must be reached for successful detonation [10].

Key parameters in EFI operation characterisation include burst time, peak current and deposited energy [11] the latter being directly related to both mass and velocity of ejected material. The flyer velocity has been demonstrated to increase with respect to initial capacitor voltage [7] and mass of material impacting the explosive has also been demonstrated to be both related to initial capacitor voltage and a key contributor in defining impulse of impact between flyer and explosive [12]. The electrical signal applied from the capacitor hence has the dual influence on both velocity and mass of ejecta. With finite energy transferable from the capacitor, these parameters of mass and velocity are inherently related.

EFI are not unique in using electrical signals to project a mass of plasma and other materials to a desired velocity; railguns demonstrate some similarity in their operation [13], though it should be noted that the plasma generated is a lower temperature and pressure owing to less confinement in railgun applications.

EFI operating under similar electrical conditions to rail guns;

C. F. Dowding is with the University of Lincoln, Brayford Pool, Lincoln, LN6 7TS, UK (e-mail: cdowding@lincoln.ac.uk). 
hence forming plasmas ejected from the unit during their operation, require consideration of the plasma-magnetic field interactions during operation.

A technique called plasma pinching is reported in [14] to shape the plasma and control the directionality of the projectile. [14] indicates that the geometry of the generating structure (in an EFI this would be the bridge) in part defines the shape of plasma formed.

A further governing factor for the geometry of the plasma generated is the magnetic field induced during current flow [15]. Where plasma expansion is constricted due to the presence of a magnetic field, such as where one is induced through large current flow, a pinching effect is observed.

The work presented in this publication seeks to build upon the work conducted on other plasma-based devices to exploit the role of plasma formation in the operation of the exploding foil initiator. When combined with previous work $[7,12]$ this contribution seeks to facilitate the optimisation of operation of EFI and initiation circuitry for a given explosive material, with design optimization being a further future avenue leading from this.

\section{Model Development}

A numerical model of the laminar structure of the EFI (including FR4 tamper layer, copper bridge and polyimide flyer and barrel layers, electrical contact pads and via used for connecting the EFI to the firing set) was developed within a numerical simulation package (CST Simulation Suite 2018.06), taking geometric dimensions from a commercially available EFI design of standard geometry [16]. The bridge geometry being symmetrical, this axis of symmetry is indicated by the solid horizontal line in all numerical modeling figures.

This geometric model facilitated the application of an electrical input signal, directly comparable with those measured in previous investigations at this contact point [7].

Using these signals, the numerical model predicted the temperature changes of the copper bridge during the electrical discharge signal.

\section{A. Electrical Boundary Conditions}

Initial electrical (boundary) conditions of current and voltage were taken from the model developed previously and validated against empirical measurements made during EFI operation using a pair of high-voltage oscilloscope probes (Tektronix, 6015a) in [12]. The electrical model developed previously [12] includes the ability to replicate the saturation effects reported in [7].

Electrical current and voltage profiles generated by the previously developed analytical model were then utilized in the numerical model, developed herein, which simulated electrical conduction and subsequent temperature increases using a coupled, time iterated EM-thermal model. The temperature rise of the preceding step was coupled back into the electrical model, influencing the electrical characteristics of the next time step in an iterative fashion.

\section{B. Numerical Modelling}

The current and voltage profiles for a range of initial capacitor voltages, as commonly used for this model of EFI, were applied through the conducting pads of the EFI in the model. The electrical signals were considered in $0.5 \mathrm{~ns}$ time intervals (to align with the sampling rate achievable by laboratory equipment; signals could not be verified over smaller time intervals) with initial temperature of 293K. A 3D electrical field monitor was placed on the EFI bridge layer.

Voltage increments of $600 \mathrm{~V}$, from $600 \mathrm{~V}$ to $3000 \mathrm{~V}$ were chosen to consider an array of initial capacitor voltages ranging from no initiation effects to saturation effects for the chosen model of EFI, as reported previously [7].

The finite element model produced a 3D thermal plot of the EFI bridge. In Figure 1 the bridge is viewed independently from the rest of the structure and the temperature is scaled from 0 to $89661.2 \mathrm{~K}$, which is the temperature equivalent to the first ionization energy of copper [17].
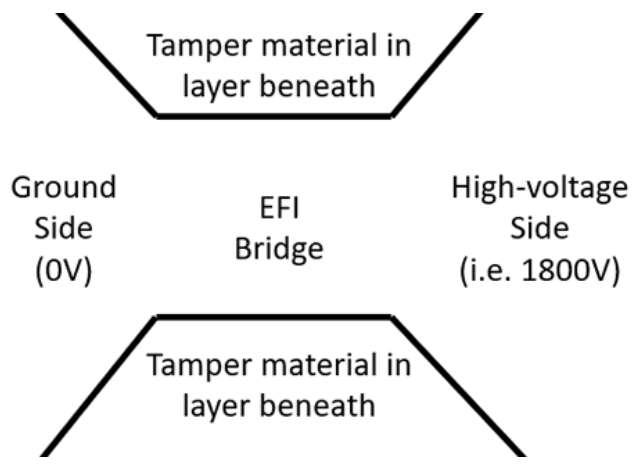

(a)

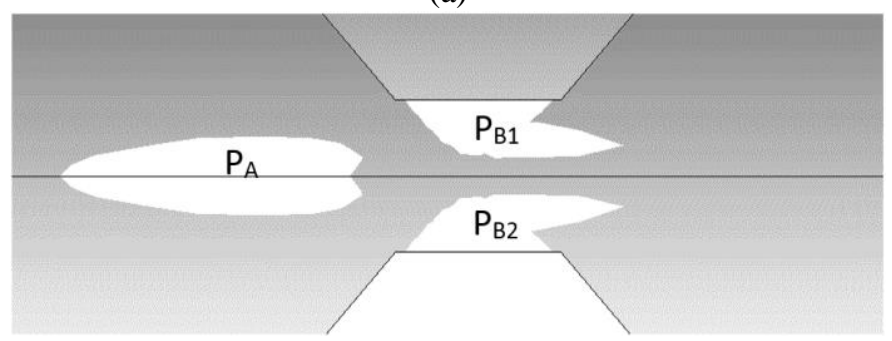

(b)

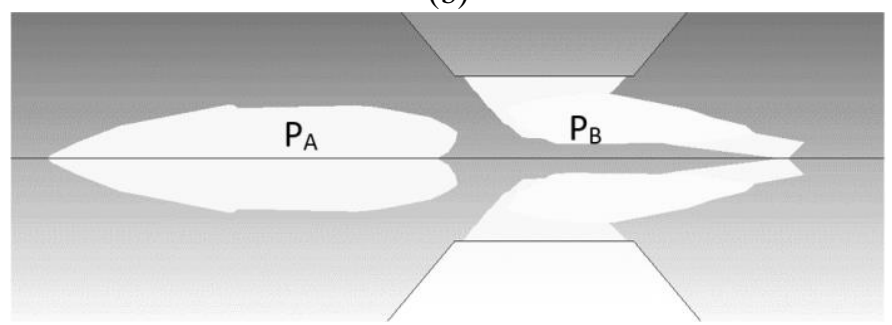

(c)

Figure 1: a) Initial EFI down barrel image with key attributes identified and temperature gradient indicating the location of the development of plasma from $b$ ) distinct regions of plasma formation and; c) final combination of plasma regions $P_{B 1}$ and $P_{B 2}$ to form region $P_{B}$. Images from above, directed down-barrel.

During the simulation, plasma material (i.e. material which has been elevated above $89661.2 \mathrm{~K}$ ) is observed as developing in distinct regions, labelled $\mathrm{P}_{\mathrm{A}}$ (ground side of the EFI) and $\mathrm{P}_{\mathrm{B}}$ 
(high-voltage side of the EFI) in Figure 1. Region $\mathrm{P}_{\mathrm{B}}$ is formed from two plasma formations that develop symmetrically, owing to the symmetry of the numerical model. Figure $1 \mathrm{~b}$ demonstrates a point early in the plasma's formation, Figure 1c shows the point at which the two symmetric regions $\mathrm{P}_{\mathrm{B} 1}$ and $\mathrm{P}_{\mathrm{B} 2}$ merge. When the two distinct regions of plasma $\left(\mathrm{P}_{\mathrm{B}}\right)$ merge it is deemed that bridge ejection commences due to high current attenuation by the plasma across the entire width of the EFI bridge allied with the magnetic field reconnection as the two plasma regions $\mathrm{P}_{\mathrm{B} 1}$ and $\mathrm{P}_{\mathrm{B} 2}$, combine. This magnetic reconnection transfers kinetic energy to the plasma, ejecting it and the flyer layer up the barrel in the top layer of the EFI's laminar structure. This sequence has been demonstrated using a magnetohydrodynamic model of EFI operation [18].

The area of material reaching copper plasma temperatures (the total mass of regions $\mathrm{P}_{\mathrm{A}}$ and $\mathrm{P}_{\mathrm{B}}$ ) is recorded allowing for the mass of ejected copper material to be calculated. The mass of copper, as predicted using the logic described herein, removed from an EFI bridge initiated with a range of initial capacitor voltages are presented in Figure 2.

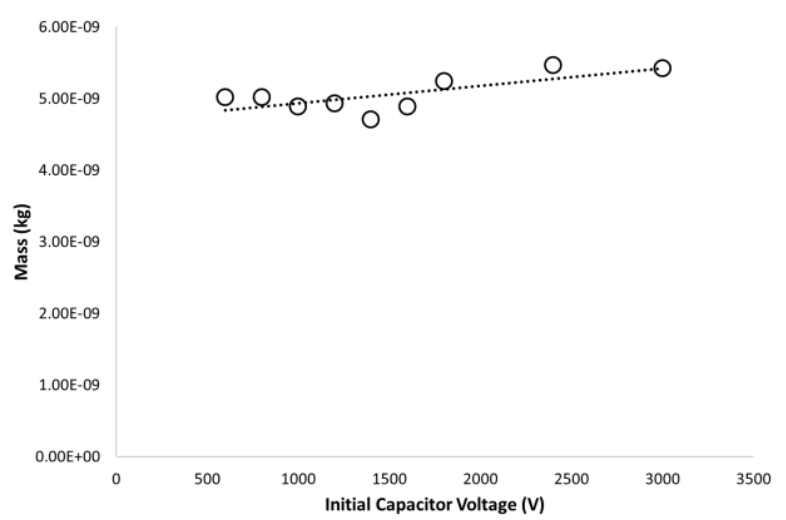

Figure 2: EFI numerical model prediction of plasma mass formed within the bridge with respect to initial capacitor voltage.

As can be seen in Figure 2, the numerical model exhibits little variance in mass ejection across the full range of initial capacitor voltages considered. The minima in predicted mass ejection in the middle of this data set is considered further in Figure 10.

The high potential differences across the EFI bridge combined with the current flow through the bridge generate a magnetic field that draws together the plasma fields forming either side of the bridge. This plasma pinching is a potential cause of the shape of the plasma regions formed [19]. Upon connection of the plasma regions, the magnetic reconnection transfers energy to kinetic energy in the plasma, ejecting it from the bridge.

To assess this, the model was altered by modifying the field which influences the pinching. A permanent magnetic field was applied to the region to combine with the effect of the magnetic field established through electron movement. This permanent magnetic field modified the previously predicted spatial distribution of both plasma formation. After modification, geometries of both regions $\mathrm{P}_{\mathrm{A}}$ and $\mathrm{P}_{\mathrm{B}}$ were altered to varying degrees dependent upon the direction and/or field strength of the additional magnetic field applied, thus verifying that plasma pinching causes the irregular shape of the plasma.

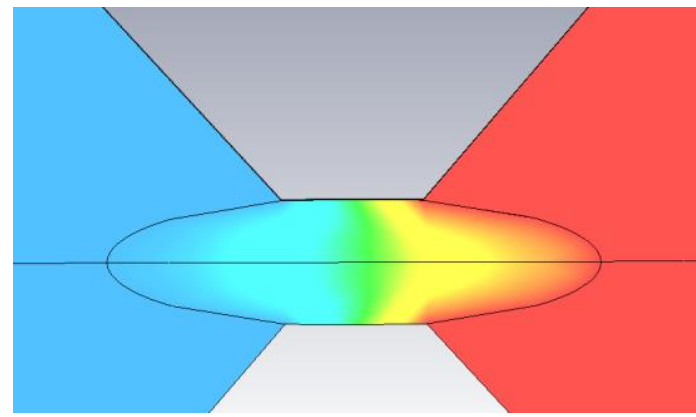

(a)

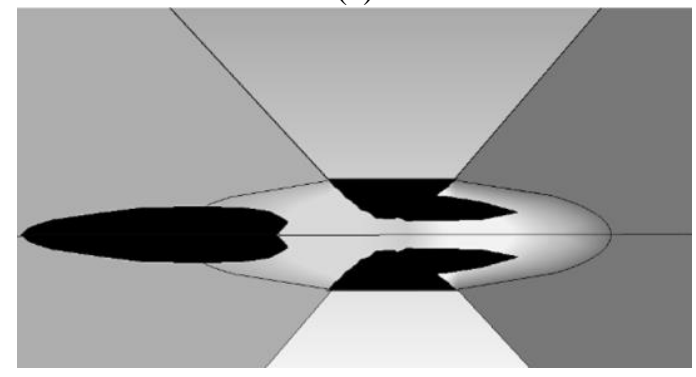

(b)

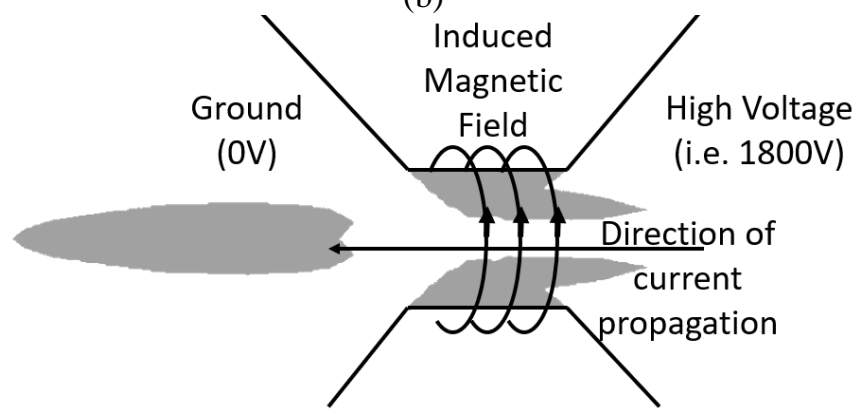

(c)

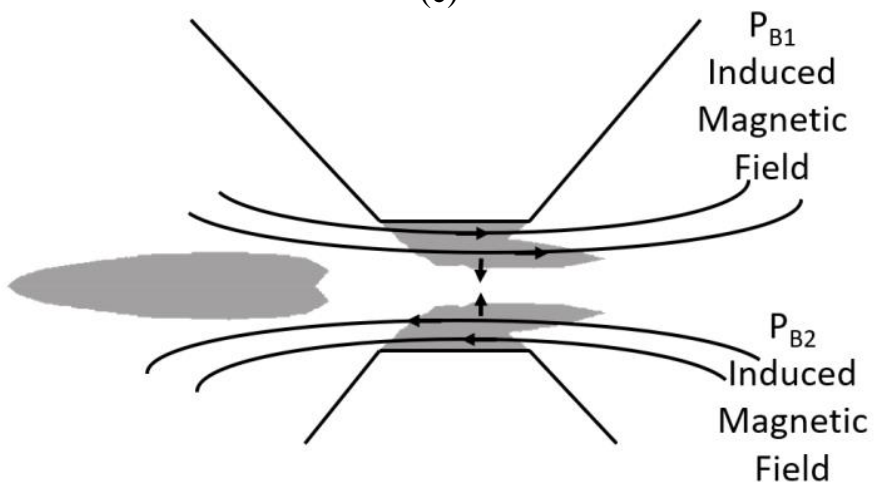

(d)

Figure 3: Electric field plotted through the EFI bridge with: a) high voltage, $1800 \mathrm{~V}$ (red) to ground, OV (blue) mapped across at a typical peak voltage for EFI operation, $b$ ) Figure 3 a with plasma formation from Figure 1 overlaid (black)

c) Schematic indicating the induced magnetic field generated during capacitor discharge through EFI bridge and d) Schematic indicating direction of plasma evolution under induced magnetic field of figure $3 c$ and further magnetic fields induced by this.

As one side of the bridge is connected to the high voltage discharge of the capacitor and the other to ground, a large 
potential difference is generated across the bridge. Modelling the electric field within such a potential difference confirms the bias of electric field intensity to one side. Moreover, this spatial offset matches the location of initial plasma generation, as can be seen in Figure 3.

Figures $3 \mathrm{c}$ and $3 \mathrm{~d}$ are schematic of the electric and magnetic fields present through the plasma formation. The high-voltage side (a typical peak voltage identified in Figure 3c) is connected a trigger vacuum gap (TVG) and the other side of the bridge to ground with a charged capacitor in parallel with TVG-EFI.

Here the traversal of electrons through the bridge between high-voltage and ground sides generates a magnetic field as indicated in figure $3 c$ [20]. This field draws the plasma regions forming closer together, creating the pinched effect and shapes the regions of plasma formation observed in Figure 1 and overlaid above in Figure 3b-d. The movement of plasma regions $\mathrm{P}_{\mathrm{B} 1}$ and $\mathrm{P}_{\mathrm{B} 2}$ towards the centre axis of the figure generates two opposing magnetic fields. Upon contact of the two plasma regions, the magnetic reconnection results in the magnetic energy being transferred to kinetic energy and the plasma being propelled in directions normal to its previous direction of propagation (into and out of the plane of the page in the case of Figure 3) [20]. The non-symmetric laminar structure of the EFI, with its tamper layer beneath the bridge, directs the ejection primarily in the one direction, i.e. up the barrel.

\section{LABORATORY MEASUREMENT}

To validate the numerical modelling, laboratory measurements were taken from EFI initiated at a range of initial capacitor voltages. As reported in [7], 600V initial capacitor voltages failed to eject bridge material. EFI initiated with initial capacitor voltages, delivered by the firing set, from $1200 \mathrm{~V}$ to $3000 \mathrm{~V}$ in $600 \mathrm{~V}$ increments were characterized.

The initiated units were analyzed using a white light interferometer (Zygo Neoscope NewView 600) with a $2.5 \mathrm{x}$ objective lens giving a maximum field of view capability of $2.81 \times 2.10 \mathrm{~mm}$. The interferometer was focused down the barrel of the EFI and set to scan through its maximum height profile of $150 \mu \mathrm{m}$ to produce surface profiles. Scanning electron micrographs from an SEM (Neoscope JCM-5000) were used to image the barrel region in order to ascertain the nature of the null data points within the white light interferometry data.

As can be seen in Figure 4, the white light interferometer identifies a region wherein the bridge remains at its original height and those regions wherein the original height is no longer reached and therefore bridge material has been removed. The region unaccounted for in Figure 4, labelled " $X$ ", is observed in Figure 5. Figure 4 clearly demonstrates correlation between the region analyzed with the two methods. Whilst the Interferometer is unable to capture some data, whether out of depth range or optical diffusion, there is good agreement in the boundaries of the region of interest. The specific area of interest is defined below.

This region (marked " $X$ " in Figures 4 and 5) was observed to be at a lower level than the original bridge material but, due to its depth, returned insufficient specular reflection for interferometric analysis. Furthermore, the increased roughness of material that has been adjacent to erupting plasma reduced specular light reflection (as required for white light interferometry).

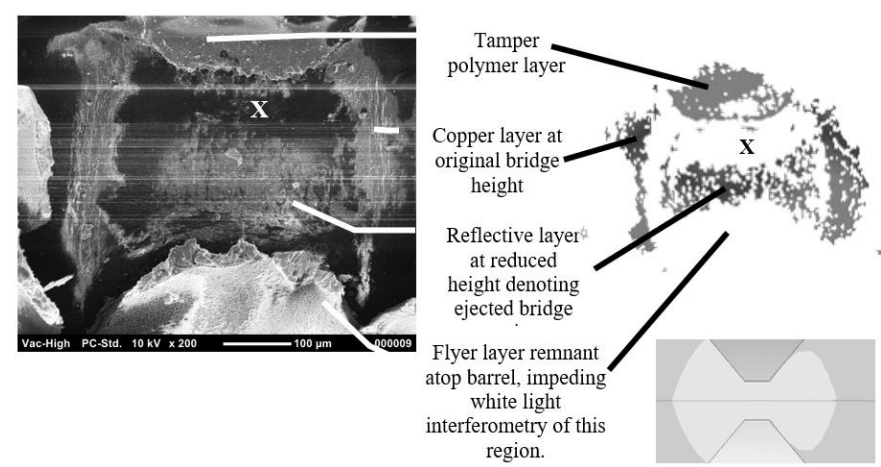

(a)

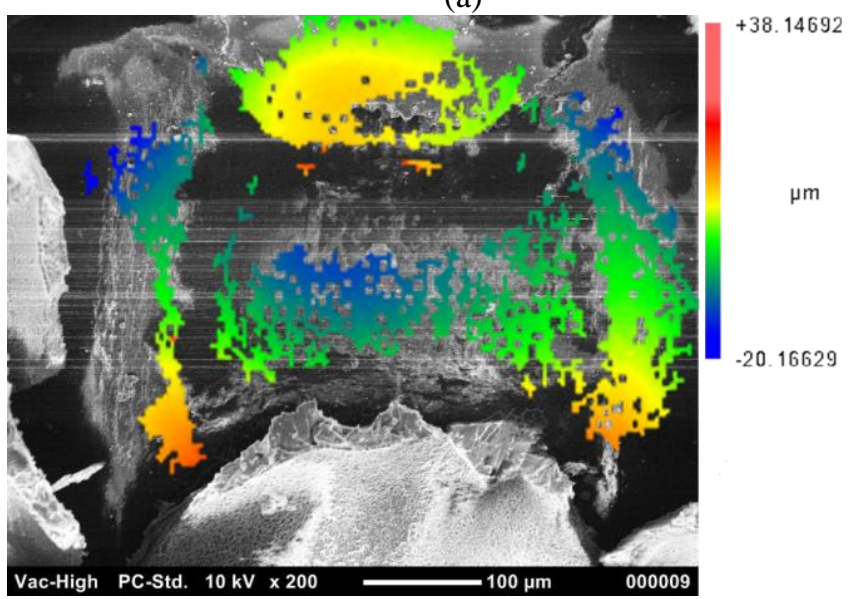

(b)

Figure 4: a) Scanning Electron Microscope and White Light Interferometer images identifying key features. (Inset) CST model of EFI bridge denoting orientation of main figures and b) Scanning Electron Microscope image of Figure 4 a with White Light Interferometer data of Figure 4 a overlaid.

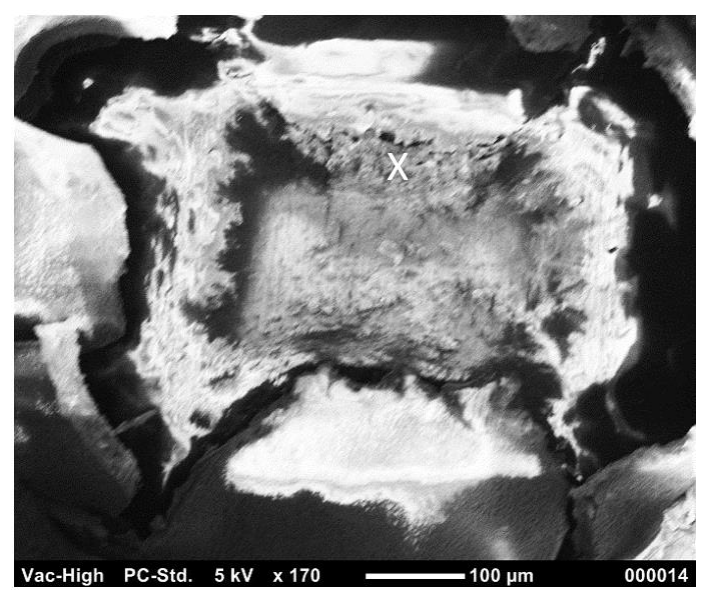

Figure 5: Increased magnification of EFI bridge using scanning electron microscope.

The area of copper bridge ejected, identified by the lower region of the surface plot where copper has been removed, was 
measured using the accompanying analysis software (MetroPro 10.0.3). The ejected region of bridge material is situated within the outlined region of Figure 6. Measurements of this region were made using the analysis tools within the interferometer software.

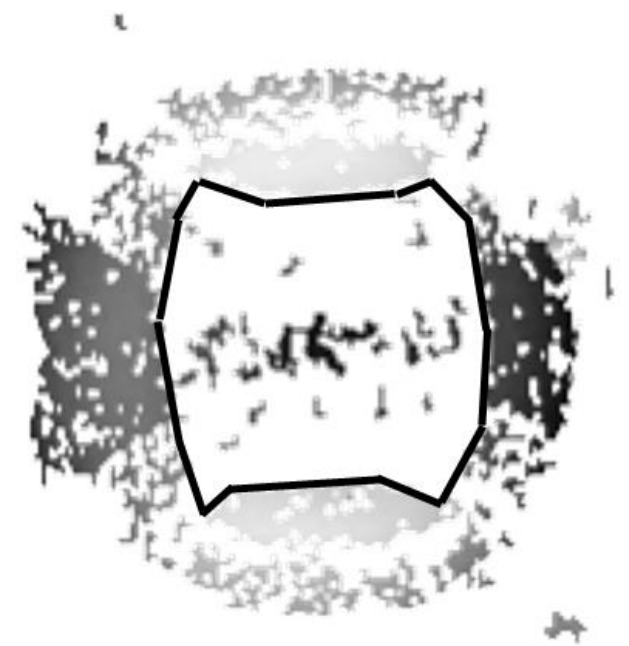

Figure 6: Surface plot of EFI down barrel region indicating area identified as that having experienced bridge removal. Black indicating lower regions, grey progressively higher regions. White areas indicating no surface detected within the scan range.

Four EFIs were initiated at each of the initial capacitor voltages and the average bridge material ejected, measured using the interferometric surface plots, was then compared with those taken from the numerical model, as shown in Figure 7.

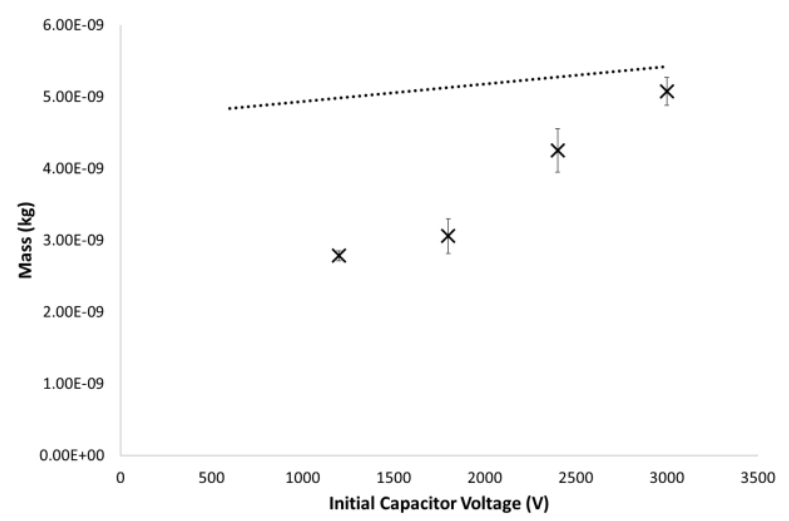

Figure 7: EFI numerical model prediction (...) of mass of plasma formed and interferometrically measured $(X)$ bridge mass ejected at a range of initial capacitor voltages.

\section{RESUlTS AND DISCUSSION}

As can be seen in Figure 7, the numerical and laboratory measurements are of the same order of magnitude; however, the trend observed differs to that of the interferometric plots. It is proposed that, for improved understanding of the mechanism of operation, the separate regions of plasma formation $\left(\mathrm{P}_{\mathrm{A}}\right.$ and $\left.\mathrm{P}_{\mathrm{B}}\right)$, their evolution and influence on current flow and material ejecta be considered.

\section{A. Plasma Region Formation}

Further analysis of the numerical model results was conducted to explore various avenues of the cause for the differing trend in mass of ejected material between numerical prediction and empirical data. This included the measurement of regions $\mathrm{P}_{\mathrm{A}}$ and $\mathrm{P}_{\mathrm{B}}$ separately at the time of ejection, as opposed to their combined total as previously graphed.

This new numerical analysis approach generated mass ejection predictions displayed in Figure 8.

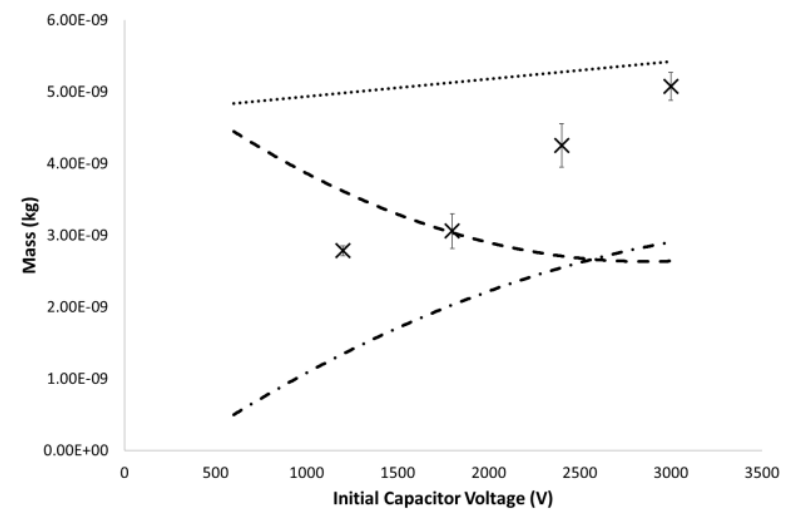

Figure 8: EFI numerical model prediction of plasma mass formed at Region $P_{A}(-\cdots)$, Region $P_{B}(--)$, the total mass of plasma formed (...) and interferometrically measured $(X)$ bridge mass ejected at a range of initial capacitor voltages.

From Figure 8 it can be seen that there is a decline in plasma formed in region $\mathrm{P}_{B}$ as capacitor voltage increases and a subsequent increase in plasma formation in region $\mathrm{P}_{\mathrm{A}}$. Region $\mathrm{P}_{\mathrm{A}}$ is located at the inlet end of the bridge (defined by the polarity of current applied), region $\mathrm{P}_{\mathrm{B}}$ (located within the bridge and expanding beyond), is downstream with the flow of current considered.

The numerical model demonstrates that an increase in electrical current flowing into the bridge forms larger plasma formations at the input region, $\mathrm{P}_{\mathrm{A}}$, hence reducing the current available for the formation of plasma taking place downstream inside the bridge itself. This change in how plasma regions are formed is explored further in 'Mode of Operation', below. The trend identified in the model correlates well with electrical signal data measured across the EFI during operation where, at $3000 \mathrm{~V}$ initial capacitor voltage, a clipping effect is seen on the current trace [7]. Based upon the numerical model presented, it is postulated that clipping is due to the development of plasma region $\mathrm{P}_{\mathrm{A}}$. This plasma formation restricted the current flow, explaining both the reduced current transfer and the earlier initiation time for higher initial capacitor voltage operation first observed in [7].

At lower initial capacitor voltages region $\mathrm{P}_{\mathrm{B}}$ is the most significant mass that forms, region $\mathrm{P}_{\mathrm{A}}$ formation is comparatively smaller and slower in development than when modelled at higher initial capacitor voltages. Indeed, at the lowest voltage simulation conducted, region $\mathrm{P}_{\mathrm{A}}$ is of negligible volume. These results align well with the laboratory measurements, collected in Section III and displayed in Figure 7 , wherein the mass predicted by the numerical model for 
region $\mathrm{P}_{\mathrm{B}}$ and that measured using the white light interferometer are in close agreement.

At higher initial capacitor voltages, the regions are more evenly distributed, region $\mathrm{P}_{\mathrm{A}}$ develops to a larger mass at a faster rate; hence, both bear equal significance. Both regions $P_{A}$ and $P_{B}$ form plasma material that is ejected. The temperature differential is reduced between these regions and the rate of plasma evolution in each of these regions is more uniform than at lower initial capacitor voltages. Therefore, at these higher voltages, it is the total plasma formed that is ejected up the barrel; this aligns well with interferometric measurement of Section III at the larger voltages. The following section considers these distinct operational modes at extreme voltages, and the take over from one to the other.

\section{B. Modes of Operation}

When considering the regions of plasma development and its evolution with respect to initial capacitor voltage, it becomes clear that there is a take-over from the $\mathrm{P}_{\mathrm{B}}$ region being dominant to an equal $\mathrm{P}_{\mathrm{A}}-\mathrm{P}_{\mathrm{B}}$ significance as voltage is increased.

Higher initial capacitor voltages, where the plasma region formation is distributed in a ratio approaching 1:1 of regions $\mathrm{P}_{\mathrm{A}}: \mathrm{P}_{\mathrm{B}}$, suggests that the total plasma mass is ejected. This boundary was chosen as it aligned with the intersection between the declining mass of region $P_{B}$ and increasing mass of region $\mathrm{P}_{\mathrm{A}}$. This high proportional volume of plasma within region $\mathrm{P}_{\mathrm{A}}$ is also believed to be the cause of saturation effects when considering current flow, as reported in [7].

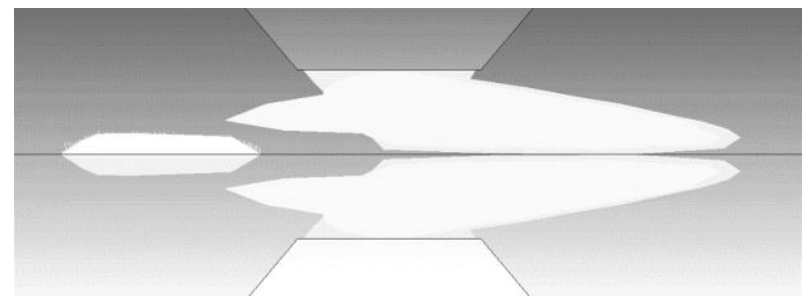

(a)

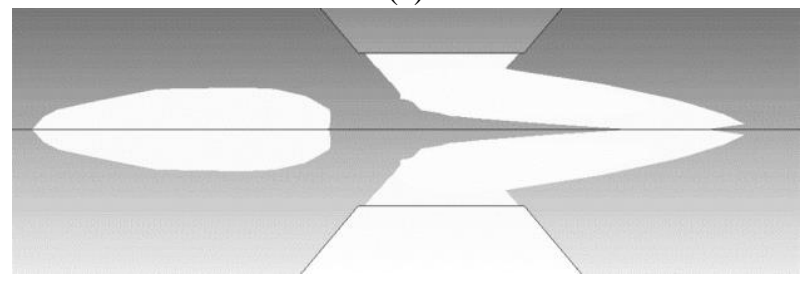

(b)

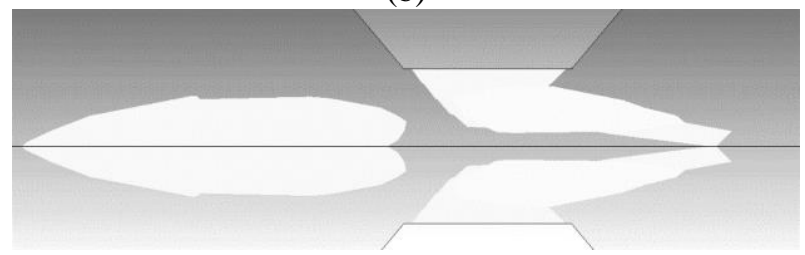

(c)

Figure 9: Numerical model predictions of plasma regions formed at point of ejection displaying the evolution of distribution from (a) $600 \mathrm{~V}$ (predominantly region $P_{B}$ ); (b) $1800 \mathrm{~V}$ (upper bound of the region $P_{B}$ dominant); and (c) $3000 \mathrm{~V}$ (both region $P_{A}$ and $P_{B}$ contributing).

If large initial capacitor voltage behavior is compared to lower initial capacitor voltages, wherein the ratio $\mathrm{P}_{\mathrm{A}}: \mathrm{P}_{\mathrm{B}}$ drops as low as 1:9, only the larger region, $P_{B}$, is ejected. The initial capacitor voltage dependent evolution of these plasma regions is displayed in Figure 9 wherein white areas denote plasma formation (i.e. temperatures greater than 89661.2K); temperatures below this are not displayed in the figures.

Applying this relationship, wherein even distribution ejects both regions and a higher weighting to region $\mathrm{P}_{\mathrm{B}}$ only ejects predominantly the latter, leads to the mass ejection prediction displayed in Figure 10, which can be assessed against interferometric measurements in Figure 10b.

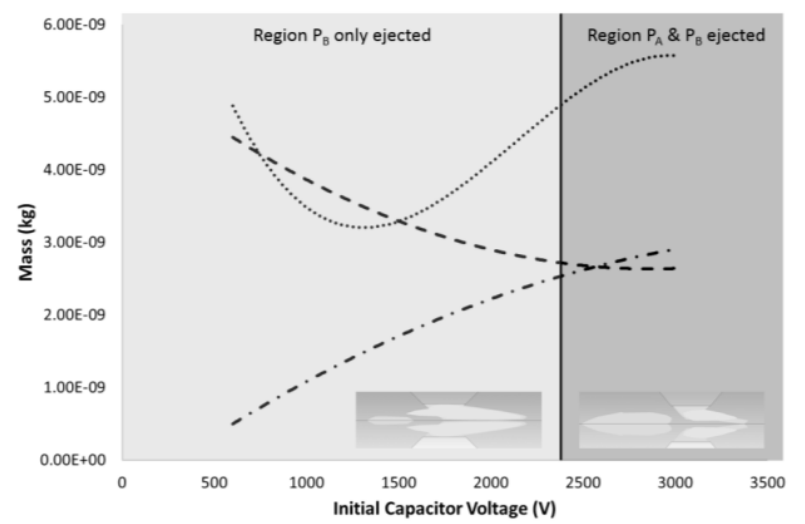

(a)

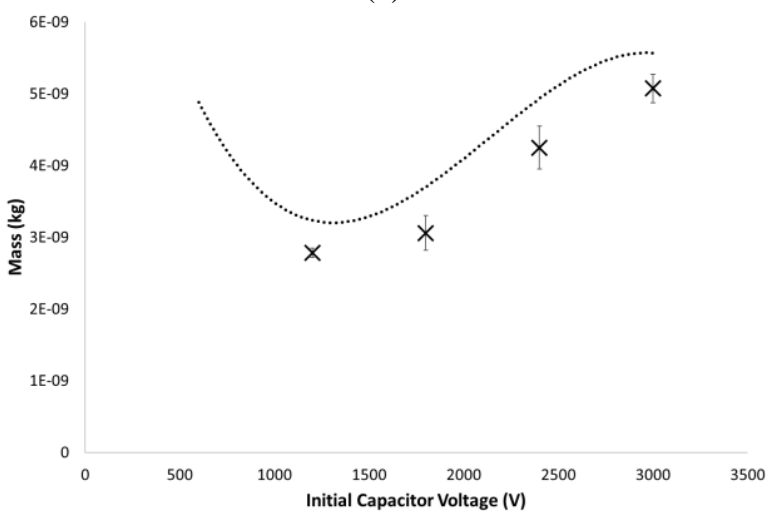

(b)

Figure 10: (a) EFI numerical model prediction of plasma mass formed at Region $P_{A}(-\cdots)$; Region $P_{B}(---)$; total mass of plasma predicted to be ejected (...). Insets of bridge plasma zones formed are provided in the relevant modes of operation of the graph. (b) Plasma mass predicted to be ejected from EFI for a range of initial capacitor voltages from both numerical modelling (..) and interferometric measurement $(X)$.

Figure 10b exhibits good correlation between mass of plasma formed in the numerical model and mass of ejected bridge measured empirically. The trend identified in Figure 10 emphasizes the non-linear relationship which was first indicated in Figure 2.

Notably the masses ejected during laboratory experimentation are consistently beneath the numerical model's predicted plasma mass. This deviation may be because not all of the mass predicted to reach plasma state in the relevant region(s) is ejected from the EFI, some of this material is instead redeposited at the bottom of the EFI barrel. This would 
make the numerical model an overestimate as observed in Figure 10b.

\section{Minimum Initiation Voltage}

By considering the time of plasma ejection predicted by the numerical model, the analytical model developed in [12] was then utilized to identify which points would result in an ejection of the EFI flyer. The $600 \mathrm{~V}$ and $800 \mathrm{~V}$ initial capacitor voltages appear to have insufficient energy to eject the bridge and flyer material atop it post-plasma formation. This owes to the reduced initial energy available within the capacitor at these lower initial capacitor voltages, the bridge geometry and the rate of plasma formed is too large and widely distributed for ejection. Plasma barely formed before the available energy supply from the capacitor was exhausted, thus the plasma formed is insufficient to provide the necessary pressure to propel the EFI flyer layer to the top of the barrel.

To validate this hypothesis, EFI were observed before and after a $600 \mathrm{~V}$ capacitor discharge through the bridge. No ejection from the barrel was observed; however, material of a mass similar to magnitudes predicted by the numerical model was observed to have changed state during the capacitor discharge. This is plotted in Figure 11.

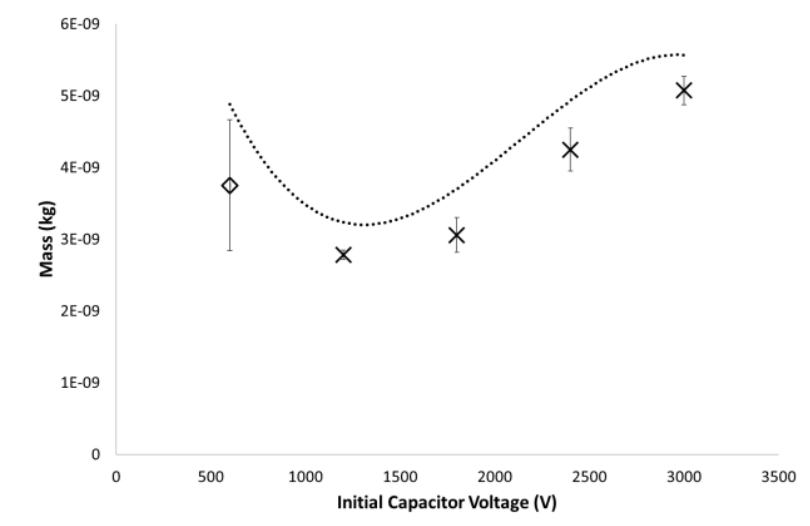

Figure 11: Figure 10 with the additional point $(\diamond)$ marking laboratory measured mass converted to plasma for a $600 \mathrm{~V}$ initial capacitor voltage discharge from the $C D C$.

Moving above $800 \mathrm{~V}$, the initial capacitor voltages begin to display reduced plasma mass and a more rapid formation, owing to proportionally larger currents channeled by the bridge geometry causing the plasma of region $\mathrm{P}_{\mathrm{B}}$ to be pinched and magnetic reconnection to occur before large plasma volumes in the $\mathrm{P}_{\mathrm{B}}$ region have formed. This has the two-fold effect of utilizing less energy to reach ejection and, as such, leaving more for the lower mass ejecta to be accelerated away from the EFI.

As initial capacitor voltage continues to increase, the mass also increases once more, region $\mathrm{P}_{\mathrm{A}}$ also being both large enough and in proximity to be ejected by the magnetic reconnection.

\section{CONCLUSIONS}

The work presented herein demonstrates the ability to predict mass of plasma formed and subsequent mass ejected from an EFI during various initial circuit conditions. The mechanisms for plasma evolution during EFI initiation have been discussed. The variable nature, resulting in a non-linear trend, of mass ejection with respect to initial capacitor voltage has been confirmed by interferometric measurement.

Moreover, the ability to accurately predict the mass ejection based only on initial electrical signals through numerical modelling has been demonstrated and validated against laboratory measurements. Alongside electrical simulation, the numerical model herein presented can function as a design aid in the development of new EFI without the need for hardware prototyping.

This work also demonstrates the increasing efficiency of the system as initial capacitor voltage is increased. Energy transferred to bridge plasma formation cannot be considered alone but in conjunction with other desired outputs such as mass and velocity of the ejected material. Considering these parameters in combination with the desired output for a given explosive pellet, this contribution may be combined with previous work to optimize design and operation of EFI and initiation circuitry for a given explosive material.

\section{REFERENCES}

[1] J. R. Stroud, "A New Kind Of Detonator - The Slapper," Lawrence Livermore National Laboratory, Livermore, 1976.

[2] H. R. Davies, D. J. Chapman, T. A. Vine and W. G. Proud, "Characterisation of An Exploding Foil Initiator (EFI) System," in SHOCK COMPRESSION OF CONDENSED MATTER 2009: Proceedings, Nashville, 2009.

[3] L. Nappert, "An Exploding Foil Initiator System," Defence Research Establishment, Valcartier, Québec, 1996.

[4] Q.-x. Zeng, J.-j. LV and M.-y. Li, "Fabrication and Testing of Metal Foil Planar Switch," Defence Technology, vol. 9, pp. 80 - 84, 2013.

[5] DuPont, "Kapton Stadard Products," DuPont, 2014. [Online]. Available: http://www.dupont.com/productsand-services/membranes-films/polyimidefilms/brands/kapton-polyimide-film/sub-brands/kaptonstandard-products.html. [Accessed 16 October 2014].

[6] J. M. Barker, "Exploding Foil Initiator using a Thermally Stable Secondary Explosive". United States of America Patent 5,431,104, 11 July 1995.

[7] A. J. Borman, C. F. Dowding, J. D. Griffiths and D. Seddon, "Exploding Foil Initiator (EFI) Modes of Operation Determined Using Down-Barrel Flyer Layer Velocity Measurement," Propellants, Explosives, Pyrotechnics, vol. 42, no. 3, pp. 318-328, 2016.

[8] A. C. Schwarz, "Shock-Initiation Sensitivity of Hexanitrostilbene (HNS)," in Seventh Symposium (International) on Detonation, 1981. 
[9] W. G. Proud, "Ignition and Detonation in Energetic Materials: An introduction," STO-EN-AVT-214, vol. 3, 2014.

[10] A. L. Brundage, "Modeling Compressive Reaction in Shock-Driven Secondary Granular Explosives," in ASME/JSME 2011 8th Thermal Engineering Joint Conference, Honolulu, Hawaii, 2011.

[11] Q. Zhang, P. Zhu, K. Wang, C. Xu, Z. Yang, R. Shen and G. Zheng, "An exploding foil overpressure actuator for multipoint synchronous initiation and mach reflection generation," Sensors and Actuators A: Physical, vol. 314, 2020.

[12] A. J. Borman, C. F. Dowding and D. Seddon, "Modelling of Exploding Foil Initiator and Related Circuitry for the Variable Mode of Operation," Journal of Defense Modelling and Simulation, 2019.

[13] J. H. Degnan, R. E. Peterkin, G. P. Baca, J. D. Beason, D. E. Bell, M. E. Dearborn, D. Dietz, M. R. Douglas, S. E. Englert, T. J. Englert, K. E. Hackett, J. H. Holmes, T. W. Hussey, G. F. Kiutti, F. M. Lehr, G. J. Marklin, B. W. Mullins, D. W. Price, N. F. Roderick and E. L. Ruden, "Compact toroid formation, compression, and acceleration," Journal Name: Physics of Fluids B, vol. 5, no. 8, 1993.

[14] V. M. Fomin, B. V. Postnikov, G. A. Sapozhnikov and V. P. Fomichev, "Multistage method for acceleration of bodies by a Railgun," in 19th International Symposium of Ballistics, Interlaken, 2001.

[15] H. Schmidt, "Formation of a Magnetic Pinch in InSb and the Possibility of Population Inversion in the Pinch," Physical Review, vol. 149, no. 564, 1966.

[16] Teledyne e2v, “Te2v Brochure," [Online]. [Accessed 25 September 2020].

[17] R. D. Harrison, Ed., Revised Nuffield Advanced Science Book of Data, Harlow, 2002.

[18] W. Neal and C. Garasi, "High fidelity studies of exploding foil initiator bridges, Part 3: ALEGRA MHD simulations," in AIP conference Proceedings 1793, 2017.

[19] J. Batra, A. C. Jaiswar, R. Valvi and T. C. Kaushik, "Experimental Investigations on Specific Energy Deposition and Burst Characteristics in Electrically Exploded Single Copper Wires," IEEE Transactions on Plasma Science, vol. 47, no. 1, pp. 596 - 602, 2019.
[20] L. G. Suttle, G. C. Burdiak, C. L. Cheung, T. Clayson, J. W. Halliday, J. D. Hare, S. Rusli, E. R. Tubman, A. Ciardi, N. F. Loureiro, J. Li, A. Frank and S. V. Lebedev, "Interactions of magnetized plasma flows in pulsed-power driven experiments," Plasma Physics and Controlled Fusion, vol. 62, 2019.

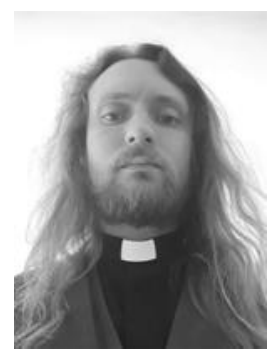

\section{Reverend Alexander J Borman} completed a BSc (Hons) OPEN degree with the Open University in 2008 and is currently completing his $\mathrm{PhD}$ at the University of Lincoln where he is also a lecturer in Mechanical Engineering.

He has a research interest in exploding foil initiator (EFI).

Orcid ID: 0000-0002-6819-8653

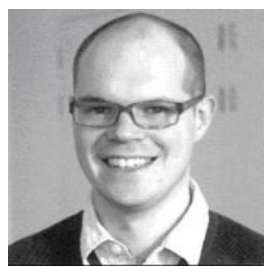

Dr Colin F Dowding completed a BEng (Hons) degree in Mechanical Engineering with the University of Loughborough in 2006 and his PhD in 2009.

$\mathrm{He}$ is currently a Senior Lecturer at the University of Lincoln with research interests in laser interactions with materials, with a specific focus on surface modification and micro-machining. Orcid ID: 0000-0002-7469-512X 